\title{
Editorial: Measurement, Modeling and Control of Different Electronic Systems
}

\author{
Attila Géczy \\ ${ }^{1}$ Guest Editor, Department of Electronics Technology, Faculty of Electrical Engineering and Informatics, Budapest University of \\ Technology and Economics (BME), H-1521 Budapest, P.O.B. 91, Hungary
}

Measurement, modeling and consequentially, precise control of electronic systems is not a new concept, but a field, which needs continuous improvement. With the evolving tools, the overall system quality and reliability improves. With the advances of instrumentations, modeling possibilities and computing power, the work of the engineers and scientists enable more efficient paths of problem solving. Our special issue focuses on these aspects.

The first paper tackles problems of open-end winding induction machines. According to a proposed backstepping control method, the tracking of the speed reference was performed with improved performance under different speed constraints. The results aim for the highest reliability in industrial applications.

The condition moitoring of nuclear power plants and various submodules, components or even cables are an important aspect of safety and reliability in the field. A review paper concentrates on the radiation multistress accelerated aging methodologies for such cables, summarizing recent results of the field. Cable reliability is important in low voltage applications too. Easing the difficulties of diagnostic testing of low voltage cables by partial discharge measurements, a presented study shows discussion of detectors, signal processing, analysis and improvement of the methodology.

Solar cells are not only important from the aspect of electron device sciences, but from the aspect of power electronics and power generation. The presented paper in the field presents an accurate and reliable photovoltaic device modeling method for a mono-crystallince solar cell. Besides computation based modeling, realized physical models of PV concepts are crucial for universities, to enable better understanding of the basic principles for the students. The following paper presents an interesting use case of a hybrid (PV-wind) power plant with storage systems in university environment.
In electric power generation, renewable energy resource aspects are becoming more important - doubly fed induction generators (DFIG) are used in many cases (such as wind systems). Selected papers focus on the specific DFIG systems. A paper present a novel approach for controlling such generators based on variable speed wind energy conversion systems. Next, doubly fed induction motors are investigated from the aspect of sensorless adaptive Fuzzy-PI speed control using a full order Extended Kalman Filter. Simulation proves a key aspect of improving robustness of the presented setup. In a following article, an optimized doubly fed induction generator control strategy is evaluated by direct torque control strategies, based on particle swarm optimization, which was found to be fast for solving nonlinear problems. An other research team also investigated the direct power control with artificial neural network-based maximum power point tracking control for variable speeds in wind energy conversion systems. Simulation is found to be essential again for improving power stability and quality. The direct torque control of a dual stator induction motor is analyzed with the help of direct and indirect matrix converters. In the comparative study, the indirect matrix converter was found to be the superior solution with simple implementation.

Modeling is found to be a key aspect in the field of electron devices as well. The recent results of low power circuit simulation is presented for lightly doped symmetrical p-channel DG MOSFET components.

To sum up, the selection of the issue provide an insight to recent advances in the field of measurement, modeling and control, in a wide range of electronic systems from large scale power engineering to the miniscule systems of electron devices. The presented cases not only give a fresh insight of the ever developing nature of our profession, but the importance of the multidisciplinary approaches applied for scientific progress. 